\title{
A RESOLVED MAP OF THE INFRARED EXCESS IN A LYMAN BREAK GALAXY AT $z=3$
}

\author{
M. P. Koprowski ${ }^{1}$, K. E. K. Coppin ${ }^{1}$, J. E. Geach ${ }^{1}$, N. K. Hine ${ }^{1}$, M. Bremer ${ }^{2}$, S. Chapman ${ }^{3}$, L. J. M. Davies ${ }^{4}$, T. Hayashino ${ }^{5}$, \\ K. K. Knudsen ${ }^{6}$, M. Kubo ${ }^{7}$, B. D. Lehmer ${ }^{8}$, Y. Matsuda ${ }^{7,9}$, D. J. B. Smith ${ }^{1}$, P. P. van der Werf ${ }^{10}$, \\ G. VIOLINO ${ }^{1}$, AND T. YAMADA ${ }^{11}$ \\ ${ }^{1}$ Centre for Astrophysics Research, University of Hertfordshire, College Lane, Hatfield AL10 9AB, UK \\ ${ }^{2}$ H.H. Wills Physics Laboratory, University of Bristol, Tyndall Avenue, Bristol BS8 1TL, UK \\ ${ }^{3}$ Department of Physics and Atmospheric Science, Dalhousie University, Halifax, NS B3H 4R2, Canada \\ ${ }^{4}$ ICRAR, The University of Western Australia, 35 Stirling Highway, Crawley, WA 6009, Australia \\ ${ }^{5}$ Research Center for Neutrino Science, Graduate School of Science, Tohoku University, Sendai, Miyagi 980-8578, Japan \\ ${ }^{6}$ Department of Earth and Space Sciences, Chalmers University of Technology, Onsala Space Observatory, SE-43992 Onsala, Sweden \\ 7 National Astronomical Observatory of Japan, Osawa 2-21-1, Mitaka, Tokyo 181-8588, Japan \\ ${ }^{8}$ Department of Physics, University of Arkansas, 226 Physics Building, 835 West Dickson Street, Fayetteville, AR 72701, USA \\ ${ }^{9}$ Graduate University for Advanced Studies, Osawa 2-21-1, Mitaka, Tokyo 181-8588, Japan \\ ${ }^{10}$ Leiden Observatory, Leiden University, P.O. Box 9513, NL-2300 RA Leiden, The Netherlands \\ ${ }^{11}$ Institute of Space and Astronautical Science, Japan Aerospace Exploration Agency, 3-1-1 Yoshinodai, 252-5210 Sagamihara, Kanagawa 252-5210, Japan \\ Received 2016 July 26; revised 2016 August 12; accepted 2016 August 15; published 2016 September 9
}

\begin{abstract}
We have observed the dust continuum of $10 z=3.1$ Lyman break galaxies with the Atacama Large Millimeter/ submillimeter Array at $\sim 450$ mas resolution in Band 7. We detect and resolve the $870 \mu \mathrm{m}$ emission in one of the targets with a flux density of $S_{870}=192 \pm 57 \mu \mathrm{Jy}$, and measure a stacked $3 \sigma$ signal of $S_{870}=67 \pm 23 \mu \mathrm{Jy}$ for the remaining nine. The total infrared luminosities are $L_{8-1000}=(8.4 \pm 2.3) \times 10^{10} L_{\odot}$ for the detection and $L_{8-1000}=(2.9 \pm 0.9) \times 10^{10} L_{\odot}$ for the stack. With Hubble Space Telescope Advanced Camera for Surveys $I-$ band imaging we map the rest-frame UV emission on the same scale as the dust, effectively resolving the "infrared excess" (IRX $\left.=L_{\mathrm{FIR}} / L_{\mathrm{UV}}\right)$ in a normal galaxy at $z=3$. Integrated over the galaxy we measure $\mathrm{IRX}=0.56 \pm 0.15$, and the galaxy-averaged UV slope is $\beta=-1.25 \pm 0.03$. This puts the galaxy a factor of $\sim 10$ below the IRX $-\beta$ relation for local starburst nuclei of Meurer et al. However, IRX varies by more than a factor of 3 across the galaxy, and we conclude that the complex relative morphology of the dust relative to UV emission is largely responsible for the scatter in the IRX- $\beta$ relation at high- $z$. A naive application of a Meurer-like dust correction based on the UV slope would dramatically overestimate the total star formation rate, and our results support growing evidence that when integrated over the galaxy, the typical conditions in high- $z$ star-forming galaxies are not analogous to those in the local starburst nuclei used to establish the Meurer relation.
\end{abstract}

Key words: dust, extinction - galaxies: high-redshift - galaxies: ISM - submillimeter: galaxies submillimeter: ISM

Supporting material: data behind figures

\section{INTRODUCTION}

It is now established that the global star formation rate (SFR) density $\left(\rho_{\mathrm{SFR}}\right)$ steadily declines beyond $z \approx 3$ following $\rho_{\mathrm{SFR}} \propto(1+z)^{-6}$ (Ellis et al. 2013; Oesch et al. 2014, 2015; Bouwens et al. 2015; McLeod et al. 2016). This "ramp up" epoch of star formation at $z \gtrsim 2$ is a new frontier of observational cosmology. However, meaningful samples of galaxies at $z>3$ have only been possible by selecting Lyman break dropouts in ultradeep optical/near-infrared imaging, resulting in rest-frame UV selected samples. Estimates of the total SFRs of these galaxies are made by correcting the UV luminosities for internal dust extinction based on the slope, $\beta$, of the UV continuum (where $f_{\lambda} \propto \lambda^{\beta}$ ). Usually this is couched in terms of the "infrared excess" (IRX), IRX $=L_{\mathrm{FIR}} / L_{\mathrm{UV}}$ (Meurer et al. 1999, hereafter MHC99), with IRX related to $\beta$ in a manner that assumes that the UV/optical photons are absorbed by interstellar dust, increasing $\beta$, are re-emitted in the far-infrared. ${ }^{12}$

\footnotetext{
12 Note that in the original definition $L_{\mathrm{FIR}}$ is the far-infrared luminosity defined by Helou et al. (1988) from IRAS bandpasses, not the integrated 8-1000 $\mu \mathrm{m}$ luminosity, as is often used in the literature, which is $\sim 50 \%$ higher for a typical dust spectrum.
}

The IRX- $\beta$ relation is useful because, at high- $z$, one typically only has a direct measurement of $L_{\mathrm{UV}}$ (usually defined as $\left.\nu L_{1600}\right)$ and $\beta$. Thus, given an uncorrected $L_{\mathrm{UV}}$ and $\beta$, it is possible to predict $L_{\mathrm{FIR}}$ and therefore the total SFR. A simple question is whether or not the same IRX- $\beta$ correction derived for local systems can be applied at high- $z$. There are several reasons for worry: (1) star formation might be proceeding in a different manner in the gas-rich disks of early galaxies compared to (comparatively) quiescent local disks and starburst nuclei (Tacconi et al. 2010; Swinbank et al. 2015), (2) the evolution of dust production at early times has not yet been established; it is certainly expected that systematic differences in the metallicity of the interstellar medium (ISM) of high- $z$ galaxies compared to local systems could result in a different dust reddening law, (3) the IR and UV emitting regions might not be spatially coincident-there could be both heavily obscured and unobscured lines of sight in the same source (Douglas et al. 2009), which will affect galaxy-averaged measurements of IRX.

It is now possible to directly detect the far-infrared dust emission of "normal" star-forming galaxies at high-z. Recent observations are revealing an interesting, but confusing, picture: Capak et al. (2015) measured the IRX for $z \sim 5$ LBGs 
Table 1

Target Properties

\begin{tabular}{|c|c|c|c|c|c|c|c|c|}
\hline ID & $\begin{array}{l}\text { R.A. } \\
\text { h m s }\end{array}$ & $\begin{array}{c}\text { Decl. } \\
\text { ०॥I, }\end{array}$ & $z_{\text {spec }}$ & $\begin{array}{c}M_{\star} \\
/ 10^{9} M_{\odot}\end{array}$ & $\begin{array}{c}L_{\mathrm{FIR}} \\
/ 10^{10} L_{\odot}\end{array}$ & $\begin{array}{c}L_{\mathrm{UV}} \\
/ 10^{10} L_{\odot}\end{array}$ & IRX & $\beta$ \\
\hline SSA22a-C40 & 221719.41 & +00 1712.7 & 2.927 & $1.0 \pm 0.7$ & $<2.9$ & $1.8 \pm 0.9$ & $<1.65$ & $-1.93 \pm 0.21$ \\
\hline SSA22a-C35 & 221720.22 & +00 1651.9 & 3.103 & $2.7 \pm 1.2$ & $<3.3$ & $3.7 \pm 1.0$ & $<0.89$ & $-1.74 \pm 0.12$ \\
\hline SSA22a-C39 & 221720.99 & +00 1708.9 & 3.076 & $1.3 \pm 0.9$ & $<2.9$ & $1.7 \pm 1.0$ & $<1.64$ & $-1.81 \pm 0.23$ \\
\hline SSA22a-C31 & 221722.89 & +001608.9 & 3.023 & $0.6 \pm 0.5$ & $<1.8$ & $3.5 \pm 1.0$ & $<0.52$ & $-2.10 \pm 0.11$ \\
\hline SSA22a-C32 & 221725.63 & +00 1612.4 & 3.301 & $1.9 \pm 1.0$ & $<4.1$ & $6.5 \pm 1.1$ & $<0.63$ & $-2.05 \pm 0.08$ \\
\hline SSA22a-C27 & 221743.06 & +00 1522.1 & 3.084 & $6.5 \pm 3.2$ & $<3.1$ & $2.6 \pm 1.0$ & $<1.19$ & $-1.03 \pm 0.32$ \\
\hline SSA22a-C36 & 221746.07 & +001643.3 & 3.066 & $5.7 \pm 2.5$ & $<3.1$ & $3.5 \pm 1.0$ & $<0.88$ & $-1.56 \pm 0.22$ \\
\hline Stack & $\ldots$ & $\ldots$ & 3.063 & $1.6 \pm 1.1$ & $1.7 \pm 0.5$ & $2.6 \pm 0.2$ & $0.65 \pm 0.21$ & $-1.96 \pm 0.12$ \\
\hline
\end{tabular}

Note. The stack consists of the nine non-detections. Upper limits are quoted at $3 \sigma$. Coordinates are Epoch J2000.

detected at far-infrared and found them to have significantly lower values than similar sources in the local universe. This indicates that the MHC99 relation may not necessarily hold at high- $z$. However, Watson et al. (2015) report the detection of dust emission from a (lensed) galaxy with a rather blue UV continuum at $z=7.5$, finding an IRX to be consistent with the local relation for the measured $\beta$.

In this Letter, we present new $870 \mu \mathrm{m}$ observations of Lyman break galaxies at $z=3$ using the Atacama Large Millimeter/submillimeter Array (ALMA). Combined with Hubble Space Telescope (HST) Advanced Camera for Surveys (ACS) imaging we resolve the IRX in a single target and thereby investigate the relative spatial extent of the stellar emission and dust absorption and the implication it has on the integrated value of the IRX. We assume a flat cosmology with $\Omega_{m}=0.3, \Omega_{\Lambda}=0.7$, and $H_{0}=70 \mathrm{~km} \mathrm{~s}^{-1} \mathrm{Mpc}^{-1}$.

\section{DATA AND OBSERVATIONS}

\subsection{Targets}

Our sample is taken from the Steidel et al. (2003) LBG redshift survey of the SSA22 field using the Palomar $5.08 \mathrm{~m}$ telescope. The optical magnitudes of the targets span $24 \leqslant R_{A B} \leqslant 26 \mathrm{mag}$, and spectroscopic redshifts have been obtained using the Low Resolution Imaging Spectrometer on Keck (Oke et al. 1995; see Table 1).

\subsection{Atacama Large Millimeter/submillimeter Array}

Coppin et al. (2015) have measured average $850 \mu \mathrm{m}$ (stacked) flux densities for LBGs at $z=3-5$, with canonically selected LBGs typically $S_{850} \approx 250 \mu \mathrm{Jy}$ at $z \approx 3$ (although with a clear mass dependence on the stacked flux density). This guided the sensitivity requirements of the follow-up ALMA Band 7 continuum observations we present here: the target $1 \sigma$ noise for a $1^{\prime \prime}$ beam was $\sigma=50 \mu \mathrm{Jy}_{\text {beam }}{ }^{-1}$.

The ALMA Band 7 observations were taken between 2014 June 30 and 2015 April 29 as a part of Cycle 2 Project \#2013.1.00362.S. The observations were split into four scheduling blocks (SBs) with each target being observed for $1073 \mathrm{~s}$. The antenna configuration delivered a resolution of approximately $500 \mathrm{mas}$ and the programme was deemed complete when the depth reached $50 \mu \mathrm{Jy}$ beam $^{-1}$. Unfortunately, this means that we are less sensitive to submillimeter emission in the LBGs if the dust is extended on scales larger than the delivered $\sim 500$ mas beam. As we will show in this section, the dust emission is resolved on this scale.

Neptune or quasar J2148+0657 were used as flux calibrators (with a 5\%-10\% uncertainty on the absolute flux calibration), and the quasar $\mathrm{J} 2148+0657$ was used as a phase calibrator for all SBs. The data were reduced and imaged using the Common Astronomy Software Application (CASA) version 4.4.0. ${ }^{13}$ Calibration involved first applying the system temperature and phase corrections and initial flagging of the autocorrelation data (shadowed and noisy antennas, channel edges, etc.). The bandpass calibration was then performed, with the phase-only gain solutions applied on-the-fly (with only central spectral channels used). This step ensures that the bandpass calibrator's temporal phase variations are correct. The bandpass solution was then applied during the gain calibrations. The resulting solutions were then visually examined, with any problematic regions flagged, and applied to the science fields.

To image the visibilities we used the CASA clean task with natural weighting to maximize the signal to noise. Only one target is detected (SSA22a-C16 at $z=3.065$ ) with a peak significance of $4 \sigma$. We show the ALMA detection in Figure 1, contoured $(2-4 \sigma)$ on the HST F814W image. The astrometry of the HST image was registered to the ground-based Subaru imaging with an rms error of 0.!2 (Hayashino et al. 2004; Matsuda et al. 2011; Yamada et al. 2012). We measure an integrated flux density of $S_{870}=192 \pm 57 \mu \mathrm{Jy}$ for the source. We stack the nine non-detections in the $u v$-plane to measure the average flux density of these sources. The stacked visibilities are imaged using same clean procedure as above, but applying a 0 ". 8 taper. We detect a $3 \sigma$ signal at the phase center with flux density $S_{870}=67 \pm 23 \mu \mathrm{Jy}$.

As can be seen in Figure 1, SSA22a-C16 has been resolved by ALMA. To confirm this, we plot the average amplitude as a function of baseline separation in Figure 2. An unresolved source has a constant amplitude for all baselines, but the data are better fit by a Gaussian profile (FWHM of 0.!94). The $\chi^{2}$ difference between the Gaussian model and a flat profile corresponds to $4.7 \sigma$.

\footnotetext{
${ }^{13}$ http://casa.nrao.edu
} 

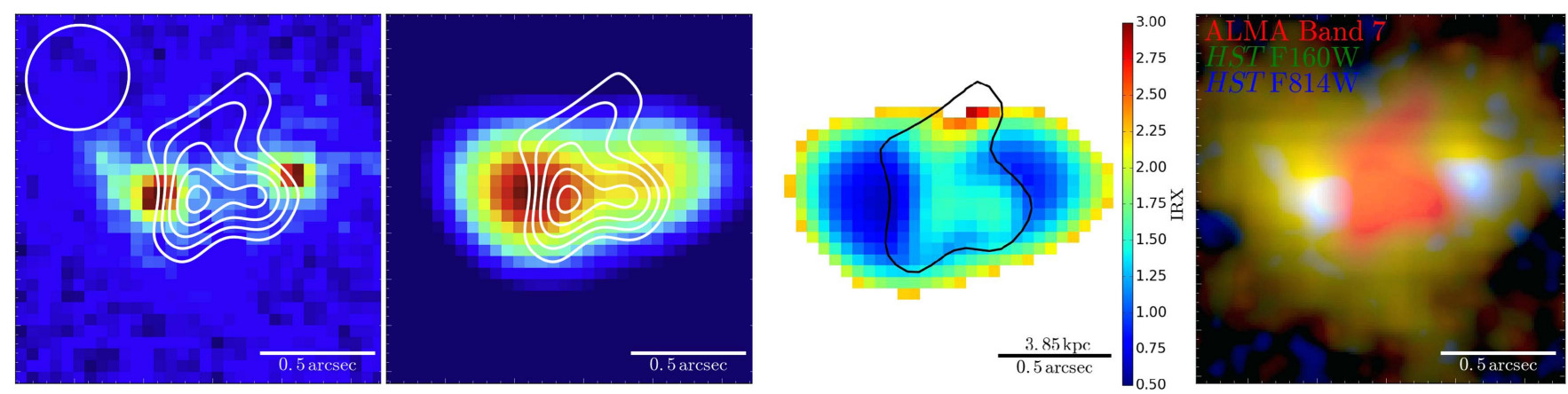

Figure 1. First panel: the ALMA $870 \mu \mathrm{m}$ contours overlaid on the HST F814W image. The contours are 2, 2.5, 3, 3.5, and 4 $\sigma$. The ALMA synthesized beam of 0 ". $46 \times 0$. " $44\left(\mathrm{PA}=-33^{\circ} .32\right)$ is shown in the top left corner. Second panel: the HST image was convolved with the ALMA synthesized beam of our observations in order to construct a resolved IRX map. Third panel: the IRX map with a pixel size of 0." 05. The ALMA flux at each pixel was translated to $L_{\mathrm{FIR}}$ using the best-fit SED from the top panel in Figure 3. Similarly, the $H S T$ flux at each pixel was translated to $L_{\mathrm{UV}}$ using the same SED. The IRX peaks at $\simeq 1.5$ and decreases to $\lesssim 0.5$ across the LBG, with values outside the contour being $2 \sigma$ upper limits. Fourth panel: the RGB plot for SSA22a-C16 with red, green, and blue channels representing ALMA Band 7, HST/WFC3 F160W, and HST/ACS F814W bands, respectively.

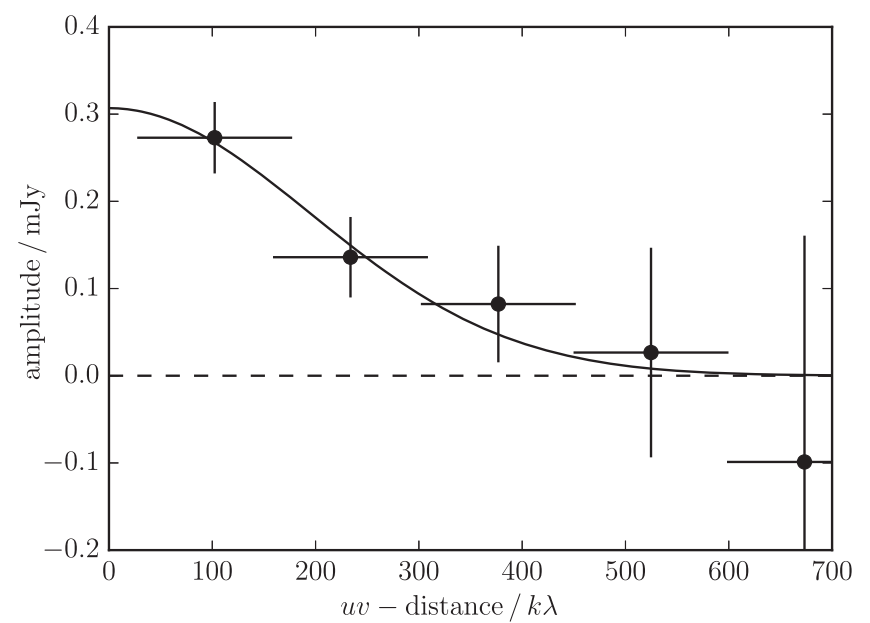

Figure 2. Average amplitude vs. $u v$-distance for SSA22a-C16, evaluated in bins of $150 \mathrm{k} \lambda$. An unresolved source has a constant amplitude for all baselines, but the data are better fit by a Gaussian (FWHM 0".94), indicating that we have resolved the dust emission in this source.

(The data used to create this figure are available.)

\section{ANALYSIS}

\subsection{Spectral Energy Distribution Fitting}

We make use of extensive multi-wavelength imaging of SSA22, including CFHT, Subaru (Hayashino et al. 2004; Matsuda et al. 2011; Yamada et al. 2012; Kubo et al. 2013), and Spitzer-IRAC (Webb et al. 2009) imaging, to obtain UV through mid-infrared photometry of the targets. We then fit the spectral energy distributions (SEDs) using CIGALE ${ }^{14}$ (Noll et al. 2009; Serra et al. 2011). We use stellar population templates from Bruzual \& Charlot (2003) with the double-burst star formation history and a Chabrier (2003) initial mass function. Extinction is implemented using Calzetti et al. (2000), and thermal dust emission uses the model of Casey et al. (2012). Since only one photometry point was available in the far-infrared, the mid-infrared power-law slope, $\alpha$, dust emissivity index, $\gamma$, and dust temperature, $T_{\mathrm{d}}$, were fixed at 2.0 , 1.6 , and $37 \mathrm{~K}$, respectively. Our choice of $T_{\mathrm{d}}=37 \mathrm{~K}$ is based on a stacked Herschel+SCUBA-2 SED of thousands of LBGs

\footnotetext{
${ }^{14}$ http://cigale.lam.fr/
}

at $z \sim 3$ from Coppin et al. (2015). In order to estimate the systematic uncertainty on $L_{8-1000}$, we (conservatively) allowed the dust emission parameters to vary between $\alpha=1.5-2.5$, $\gamma=1.2-2.0$, and $T_{\mathrm{d}}=27-47 \mathrm{~K}$ to include more extreme sources (e.g., Saintonge et al. 2013). This resulted in the additional systematic uncertainty of $0.14 \mathrm{dex}$ in the integrated infrared luminosity. The derived physical properties are summarized in Table 1. The best-fit SED for SSA22a-C16 is shown in the top panel of Figure 3. For the ALMA nondetections, we averaged the UV-mid-IR photometry and fit the SED in the same way using the stacked ALMA flux. The corresponding best fit for the "average" LBG is shown in the bottom panel of Figure 3. It is interesting to note that the average mass of the ALMA non-detected LBGs, $M_{\star}=(1.61 \pm 1.08) \times 10^{9} M_{\odot}$, is a factor 20 lower than that of the LBG we directly detected with ALMA$M_{\star}=(3.16 \pm 0.36) \times 10^{10} M_{\odot}$.

\subsection{Infrared Excess}

We estimate the SFR for SSA22a-C16, following Madau \& Dickinson (2014), with $\mathrm{SFR}_{\mathrm{UV}}=\mathcal{K}_{\mathrm{UV}} \times L_{1500}$ and $\mathrm{SFR}_{8-1000}=\mathcal{K}_{8-1000} \times L_{8-1000}, \quad$ where $\quad \mathcal{K}_{\mathrm{UV}}=1.15 \times$ $10^{-28} M_{\odot} \mathrm{yr}^{-1}\left(\mathrm{erg} \mathrm{s}^{-1} \mathrm{~Hz}^{-1}\right)^{-1} \quad$ and $\quad \mathcal{K}_{8-1000}=4.5 \times$ $10^{-44} M_{\odot} \mathrm{yr}^{-1}\left(\mathrm{erg} \mathrm{s}^{-1}\right)^{-1}, \quad$ with $\quad \nu L_{1500}=(8.8 \pm 0.3) \times$ $10^{10} L_{\odot}$ and $L_{8-1000}=(8.4 \pm 2.3) \times 10^{10} L_{\odot} \cdot{ }^{15} \mathrm{We}$ find $\mathrm{SFR}_{U V}=19 \pm 1 M_{\odot} \mathrm{yr}^{-1}$ and $\mathrm{SFR}_{8-1000}=15 \pm 4 \mathrm{M}_{\odot} \mathrm{yr}^{-1}$, giving a total $\mathrm{SFR}=34 \pm 4 \mathrm{M}_{\odot} \mathrm{yr}^{-1}$. Recall that IRX $\equiv$ $L_{\mathrm{FIR}} / \mathrm{L}_{\mathrm{UV}}$, with $L_{\mathrm{FIR}}=(4.8 \pm 1.3) \times 10^{10} L_{\odot}=(0.57 \times$ $L_{8-1000}$ ). The corresponding infrared excess is thus (IRX) $=$ $0.56 \pm 0.15$, and the UV slope (evaluated by fitting the continuum slope of the best-fit SED over rest-frame $1250-2500 \AA$ ) is $\beta=-1.25 \pm 0.03$. Thus, we can place this galaxy in context with other systems at low- and high- $z$ by placing it on the IRX- $\beta$ plot (Figure 4). Averaged over the galaxy it can be seen that our source falls significantly below the MHC99 IRX relation. The stacked detection (and individual upper limits) is more consistent with MHC99, although they are generally bluer and there is still clearly a high degree of scatter in IRX for a fixed $\beta$. One route to understanding the origin of the scatter in IRX- $\beta$ is to use our

\footnotetext{
${ }^{15}$ The Madau \& Dickinson (2014) calibration of SFR $_{\mathrm{UV}}$ is for $1500 \AA$. However, note that the MHC99 definition of the IRX uses $1600 \AA$.
} 


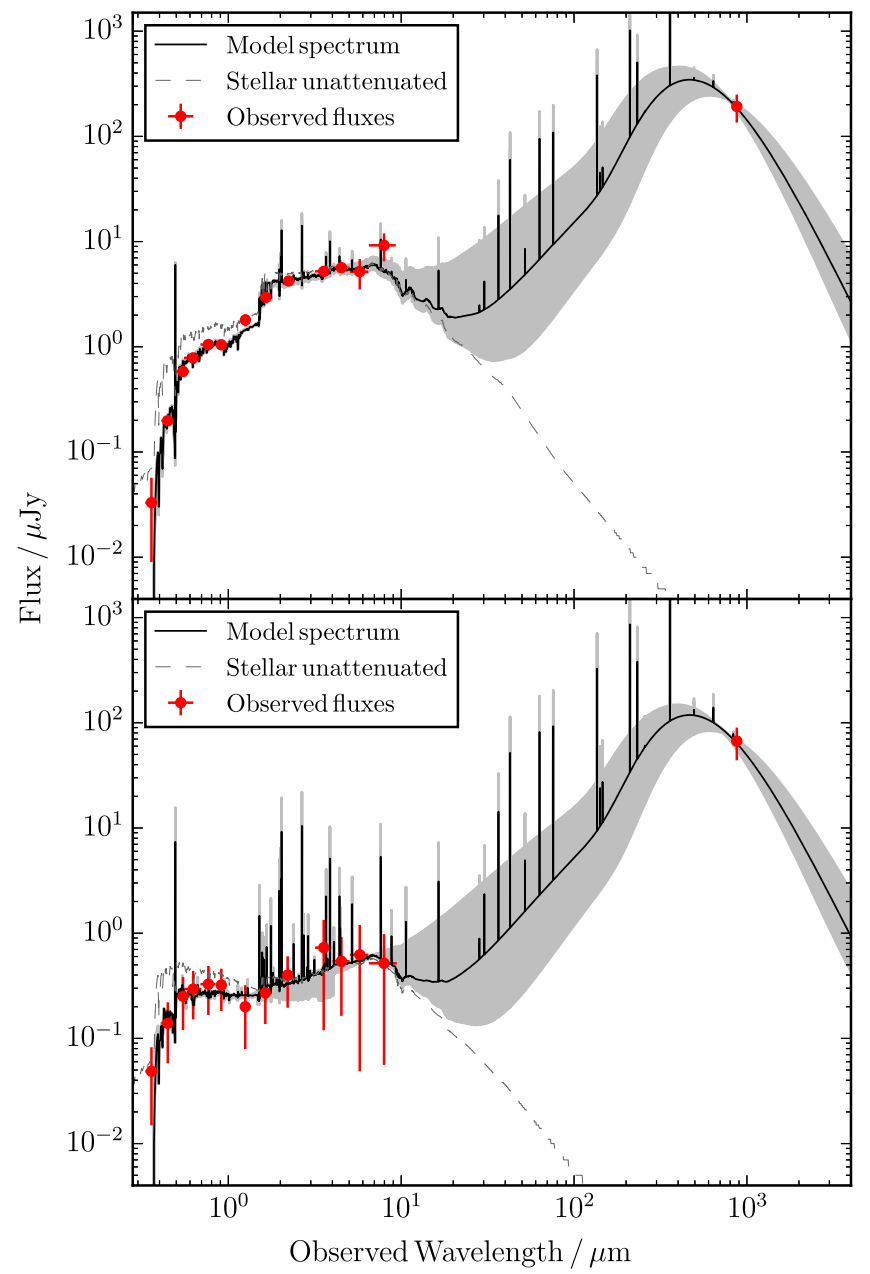

Figure 3. Top: the best-fit SED from cigale for SSA22a-C16 at $z=3.065$, with reduced $\chi^{2}=0.49$ (black solid line), resulting in in integrated IR luminosity $L_{8-1000}=(8.4 \pm 2.3) \times 10^{10} L_{\odot}($ with an additional systematic uncertainty of 0.14 dex; see the text for details). Bottom: the best-fit SED for the stacked nondetections, giving an average $L_{8-1000}=(2.9 \pm 0.9) \times 10^{10} L_{\odot}$. The errors on the photometry represent the standard deviation of the photometry values for the nine galaxies in the stack.

resolved detection to explore the variation of IRX within a single source.

Since we resolve both the rest-frame FIR and UV continuum emission, we can construct a coarse map of IRX. To do this, we grid the ALMA and HST images to the same scale and convolve the HST image with the ALMA synthesized beam to match it to the lower ALMA angular resolution (we have confirmed that the $H S T /$ ACS point-spread function is negligible compared to the ALMA beam). Individual pixel fluxes are converted to IR and UV luminosities using the best-fit (galaxy-integrated) SED as a scale. The ratio of the luminosity maps defines the IRX map, and we show this in Figure 1. In regions of the ALMA map without significant submillimeter emission but significant optical emission, the IRX derived is an upper limit. The submillimeter emission sits at the "saddle" between two clumps of bright UV emission, with the overall morphology resembling a coalescing merger or chain galaxy. Whatever its nature, it is clear that IRX varies strongly across the source (visualized by a gray rectangle in Figure 4), with at least a factor of 3 variation from the highly obscured peak of submillimeter emission to the bright knots of UV emission. An important caveat is that we do not have a resolved map of $\beta$ (the F814W band image is our only high-resolution optical image, with the $\beta$ evaluated from the seeing-limited photometry). Resolving $\beta$ would be informative because it would allow us to determine which optical "component" is dominating the galaxy-averaged UV slope.

Finally, to quantify the impact the HST-ALMA relative astrometric uncertainty of $0 . ! 2$ has on the pixel-to-pixel variations of the IRX, we performed a simple Monte Carlo simulation. We varied the relative positions of the HST and ALMA maps 1000 times by shifting the phase center of the ALMA map by a random offset sampled from a Gaussian distribution with $\sigma=0$ ". 2 . For each of the 1000 realizations, the IRX map was constructed in the same way as the "true" observations, and we take the standard deviation of the IRX range measured at the same position as the "real" map to be the $1 \sigma$ systematic error; we depict this in Figure 4 by a gray open rectangle.

\section{DISCUSSION AND SUMMARY}

As noted by MHC99, a major caveat in the application of the "standard" IRX- $\beta$ relation to high- $z$ star-forming galaxies is the assumption that they are analogous to local starbursts (the original sample for which IRX- $\beta$ was derived consists of starburst nuclei, starburst rings, blue compact dwarfs, and blue compact galaxies). These systems are characterized by compact star-forming regions in which the starlight is well described by a single stellar population. The dust responsible for obscuring this light is reasonably co-located with the young stars, and so the UV slope is directly associated with the re-radiated thermal dust emission, even assuming a simplistic screen approximation for the dust geometry. In high- $z$ star-forming galaxies, the situation is likely to be different: star formation is likely to be more clumpy and widely distributed, driven by interactions and mergers as well as the potentially unstable nature of turbulent gas-rich disks (Ivison et al. 2011; Casey et al. 2014; Simpson et al. 2015; Rujopakarn et al. 2016). This can result in a complex geometry for the dust with respect to the stars, and so when considering galaxy-integrated properties it is important to note that the shape of the stellar SED is determined by a mix of stellar populations with potentially strongly differentiated extinction.

This appears to be the situation here; the morphology of the rest-frame UV light and the infrared emission are clearly different (in fact, the LBG could be undergoing a merger; see Hine et al. 2016). Although the galaxy has approximately equal amounts of obscured and unobscured star formation, our resolved map of IRX shows that there are regions that are quite obscured $(\mathrm{IRX} \simeq 1.5)$ and regions that are relatively unobscured (IRX $\lesssim 0.5)$. This scatter in a single system predicts a large scatter in the unresolved IRX- $\beta$ for typical star-forming galaxies at high- $z$ for two reasons: (1) if dust and stars are not well mixed, as in this example, then random orientation will play an important role in the observed galaxy-averaged IRX and $\beta$, typically biasing toward higher IRX and redder $\beta$; and, related, (2) short dynamical times could potentially affect the observed IRX- $\beta$ on similar timescales, again driving scatter. Therefore, if a single IRX- $\beta$ correction is applied (regardless if it is consistent with MHC99), this large scatter must be considered as an important uncertainty in total SFR estimates if galaxy-integrated values are considered. With this in mind, the question therefore is what the appropriate IRX- $\beta$ correction is for high- $z$ star-forming galaxies? Our galaxy-averaged 


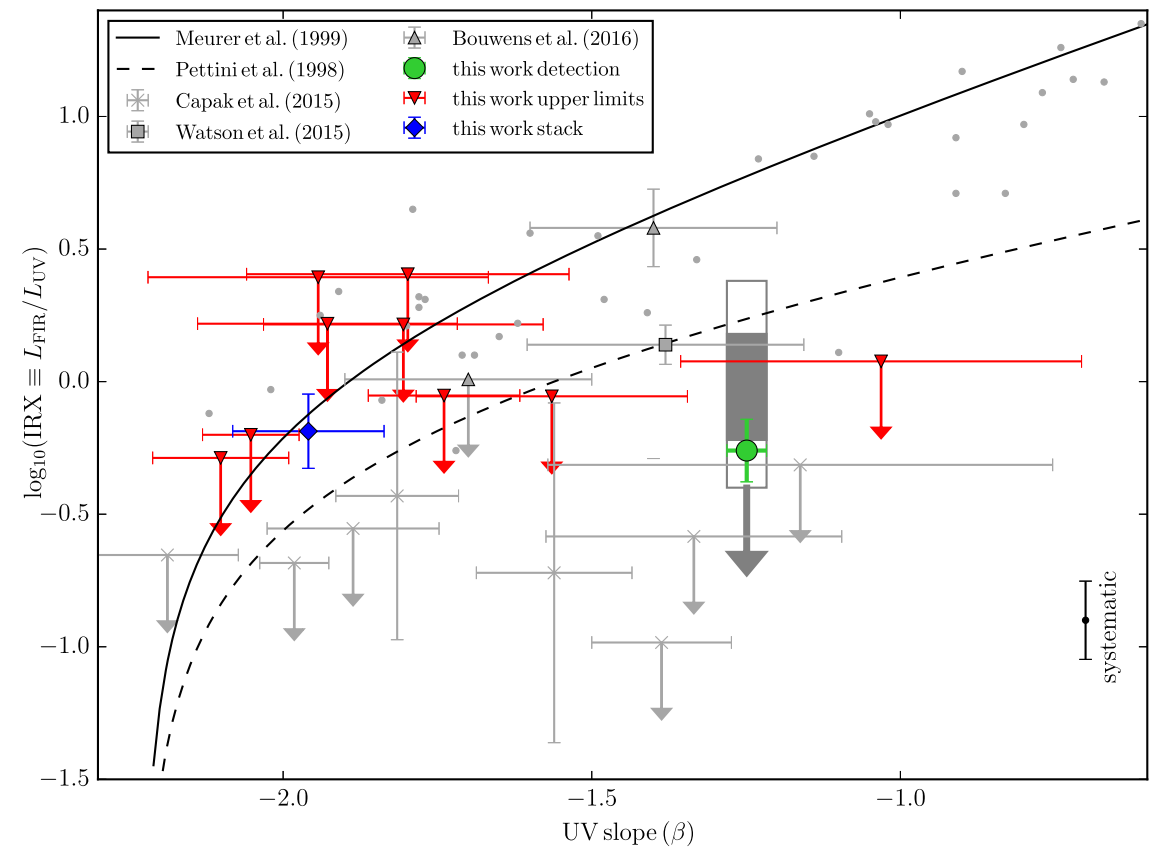

Figure 4. IRX as a function of the UV slope $\beta$. Our results are compared with that of Capak et al. (2015) at $z=5-6$, Watson et al. (2015) at $z=7.5$, Bouwens et al. (2016) at $z \simeq 2-3$, and the original Meurer et al. (1999) local sample (with $3 \sigma$ upper limits). The lines show the IRX- $\beta$ model of Meurer et al. (1999) for a Calzetti dust law (solid) and SMC-like dust law (Pettini et al. 1998; dashed). The gray filled rectangle depicts the range in IRX measured across our detection (third panel of Figure 1), the gray open rectangle shows the $1 \sigma$ systematic uncertainty coming from the relative HST-ALMA astrometry uncertainty of 0 ". 2 , and we also indicate the systematic uncertainty coming from a range of thermal dust emission models. High- $z$ results are clearly highly scattered and often consistent with very low IRX values. Our resolved map shows that IRX can vary by at least a factor of 3 in a single source, hinting that much of the scatter in the IRX- $\beta$ can be attributed simply to complex relative dust/stellar distributions in star-forming galaxies. A picture is emerging that the starburst nuclei and compact star-forming galaxies used to derive the MHC99 relation sample are poor analogs of the galaxy-integrated values of IRX at high- $z$. Finally, note that, to be consistent with MHC99, we have corrected all points to $L_{\mathrm{FIR}} / L_{\mathrm{UV}}$, by converting $L_{8-1000}$ or $L_{3-1100}$ (depending on the sample used) to $L_{\mathrm{FIR}}$, which typically corresponds to a factor of $0.4-0.5$.

(The data used to create this figure are available.)

measurements show that the LBG is not consistent with the MHC99 relation, even when adapted for an SMC-like reddening law.

Bearing in mind the factors described above, evidence is mounting that high- $z$ star-forming galaxies have systematically lower IRX for a fixed $\beta$ than observed for local starburst nuclei (see Figure 4). One explanation put forward, beyond simple geometry arguments, is the nature of interstellar dust at early epochs. At a fundamental level, dust must build up in the ISM over time, such that galaxies at $z \approx 5$ have had less then $1 \mathrm{Gyr}$ to enrich their ISM. Interestingly, Watson et al. (2015) present an ALMA detection of a $z=7.5$ gravitationally lensed LBG with implied IRX $=1.4 \pm 0.3$, showing that substantial dust reservoirs must be accumulating rather quickly in the first galaxies. It seems unlikely therefore that systematic offsets from the local relation are driven purely by metal abundance. However, without a firmer understanding of both the extinction law and the typical joint dust and UV morphology of a larger sample, typical high- $z$ star-forming galaxies it will be difficult to disentangle this.

Regardless of the details governing IRX on galactic scales, with direct measurements of both UV and infrared components in high- $z$ galaxies, there is growing evidence that the standard MHC99 IRX $-\beta$ relation will typically overestimate the total SFRs of galaxies for a given $\beta$, and this should be an important consideration for any assessment of volume-averaged SFRs based on UV luminosity functions alone. This issue will only be truly resolved with a more extensive survey of the joint IR and UV emission of a large unbiased sample of UV-selected high- $z$ galaxies; a goal we have demonstrated ALMA can achieve.

We thank the referee for a constructive report. We also thank George Bendo, UK ARC node, James Dunlop, Ian Smail, Michał Michałowski, and Rychard Bouwens for their useful contributions. K.E.K.C., M.P.K., and N.K.H. acknowledge support from the UK's Science and Technology Facilities Council (grant numbers ST/M001008/1 and ST/K502029/1). J.E.G. is supported by the Royal Society. K.K. acknowledges support from the Swedish Research Council and the Knut and Alice Wallenberg Foundation. This Letter makes use of the following ALMA data: ADS/JAO.ALMA\#2013.1.00362.S. ALMA is a partnership of ESO (representing its member states), NSF (USA) and NINS (Japan), together with NRC (Canada), NSC and ASIAA (Taiwan), and KASI (Republic of Korea), in cooperation with the Republic of Chile. The Joint ALMA Observatory is operated by ESO, AUI/NRAO and NAOJ.

\section{REFERENCES}

Bouwens, R., et al. 2016, arXiv:astro-ph/1606.05280 Bouwens, R. J., et al. 2015, arXiv:astro-ph/1506.01035 Bruzual, G., \& Charlot, S. 2003, MNRAS, 344, 1000

Calzetti, D., Armus, L., Bohlin, R. C., et al. 2000, ApJ, 533, 682 Capak, P. L., et al. 2015, Natur, 522, 455

Casey, C. M., Scoville, N. Z., Sanders, D. B., et al. 2014, ApJ, 796, 95

Casey, C. M., et al. 2012, ApJ, 761, 140

Chabrier, G. 2003, ApJL, 586, L133

Coppin, K. E. K., et al. 2015, MNRAS, 446, 1293 
Douglas, L. S., Bremer, M. N., Stanway, E. R., Lehnert, M. D., \& Clowe, D. 2009, MNRAS, 400, 561

Ellis, R. S., et al. 2013, ApJL, 763, L7

Hayashino, T., et al. 2004, AJ, 128, 2073

Helou, G., Khan, I. R., Malek, L., \& Boehmer, L. 1988, ApJS, 68, 151

Hine, N. K., Geach, J. E., Alexander, D. M., et al. 2016, MNRAS, 455,2363

Ivison, R. J., Papadopoulos, P. P., Smail, I., et al. 2011, MNRAS, 412, 1913

Kubo, M., et al. 2013, ApJ, 778, 170

Lawrence, A., et al. 2007, MNRAS, 379, 1599

Madau, P., \& Dickinson, M. 2014, ARA\&A, 52, 415

Matsuda, Y., et al. 2011, MNRAS, 410, L13

McLeod, D. J., McLure, R. J., \& Dunlop, J. S. 2016, MNRAS, 459, 3812

Meurer, G. R., Heckman, T. M., \& Calzetti, D. 1999, ApJ, 521, 64

Noll, S., Burgarella, D., Giovannoli, E., et al. 2009, A\&A, 507, 1793
Oesch, P. A., Bouwens, R. J., Illingworth, G. D., et al. 2015, ApJ, 808, 104 Oesch, P. A., et al. 2014, ApJ, 786, 108

Oke, J. B., et al. 1995, PASP, 107, 375

Pettini, M., Kellogg, M., Steidel, C. C., et al. 1998, ApJ, 508, 539

Rujopakarn, W., Dunlop, J. S., Rieke, G. H., et al. 2016, ApJ, submitted (arXiv:1607.07710)

Saintonge, A., et al. 2013, ApJ, 778, 2

Serra, P., Amblard, A., Temi, P., et al. 2011, ApJ, 740, 22

Simpson, J. M., et al. 2015, ApJ, 799, 81

Steidel, C. C., Adelberger, K. L., Shapley, A. E., et al. 2003, ApJ, 592, 728

Swinbank, A. M., et al. 2015, ApJL, 806, L17

Tacconi, L. J., et al. 2010, Natur, 463, 781

Watson, D., Christensen, L., Knudsen, K. K., et al. 2015, Natur, 519, 327

Webb, T. M. A., Yamada, T., Huang, J. S., et al. 2009, ApJ, 692, 1561

Yamada, T., Nakamura, Y., Matsuda, Y., et al. 2012, AJ, 143, 79 